Case Report

\title{
Pigmented Low Grade Malignant Eccrine Spiradenoma of the Eyelid. Unusual Presentation of a Rare Tumor. A Case Report and Review of Literature
}

\author{
Amita $\mathrm{K}^{1}$, Vijayshankar $\mathrm{S}^{2}$ and Shobha $\mathrm{R}^{3}$ \\ Department of Pathology Adichunchanagiri Institute of Medical Sciences B.G.Nagara, Mandya district \\ Karnataka state, India 571448
}

Correspondence should be addressed to: Amita K; dramitay@rediffmail.com

Received date: 5 December 2013; Accepted date: 4 March 2014; Published date: 16 October 2015

Academic Editor: Steffen Heegaard

Copyright (C) 2015. Amita K, Vijayshankar S and Shobha R. Distributed under Creative Commons CC-BY 4.0

\begin{abstract}
Objective: Malignant adnexal tumors are rare and account for $0.005 \%$ of all the skin tumors. Malignant eccrine spiradenoma is a rare adnexal tumor occurring in the extremities and trunk. Rarely has it been report in the head with only two cases described in the eyelid. In addition to this, there has been no report on the pigmented type. Case report: We report a rare case of a 70 year old female with history of pigmented nodule over the right eye since 5 to 6 years. Clinically a diagnosis of malignant melanoma was made. Cheek rotation flap with excision was done. Final histopathological diagnosis of pigmented low grade malignant eccrine spiradenoma was made. Conclusion: Pigmented malignant eccrine spiradenoma is an extremely rare tumor and should be considered in the differential diagnosis of pigmented nodules of the eyelid.
\end{abstract}

Key words: Pigmented, Eyelid, Eccrine spiradenoma, Malignant

\section{Introduction}

Most common malignant tumor of the eyelid is basal cell carcinoma accounting for $85 \%$ of the tumors followed by, squamous cell carcinoma, sebaceous carcinoma and malignant melanoma $(<1 \%)$. [Cook BE and Bartley GB, 2001]

Malignant adnexal tumors are as such rare and account for $0.005 \%$ of all the skin tumors. [Marenda SA and Otto RA, 1993] Malignant eccrine spiradenoma (MES) is a distinct but unusual adnexal tumor occurring predominantly in the extremities and trunk.
Rarely does it occur in the head and neck region. [Beekley AC et al., 1999]

Extensive pigment deposition in MES is not a recognized entity. Practically there is a need to differentiate this tumor from other malignant pigmented tumors of the eyelid like malignant melanoma, pigmented squamous cell carcinoma and pigmented basal cell carcinoma, in view of the different treatment approach in each case.

\section{Case Report}

A 70 year old female presented with history of a nodule over right eye since 5 to 6 years. To

Cite this Article as: Amita K, Vijayshankar S and Shobha R (2015), "Pigmented Low Grade Malignant Eccrine Spiradenoma of the Eyelid. Unusual Presentation of a Rare Tumor. A Case Report and Review of Literature", International Journal of Case Reports in Medicine, Vol. 2015 (2015), Article ID 658964, DOI: 10.5171/2015. 658964 
start with, initially the wound was small in size of approximately $0.5 \times 0.5 \mathrm{~cm}$. Patient noticed sudden increase in the size of swelling to the present size of $3 \times 2.5 \times 1 \mathrm{~cm}$ (Figure 1). History of bleeding from the nodule was elicited. There was no history of trauma, pain or itching at the site of wound. There was a history of diminution of vision in both the eyes since 6 years. On local examination right eye showed an ulceroproliferative fungating growth measuring $3 \times 2.5 \times 1 \mathrm{~cm}$ (Figure 1). The lesion showed brown discoloration. The lesion was seen in the right lower eyelid near the eyelash and extending up to the lateral border of the lower palpeberal conjunctiva. Floor of the ulcer was indurated and covered with slough and purulent discharge. Conjunctiva showed congestion. Cornea revealed opacity in both eyes with corneal degeneration. Right sided anterior chamber was shallow while left sided anterior chamber was unremarkable. Similarly right sided pupil reacted sluggishly to the light.

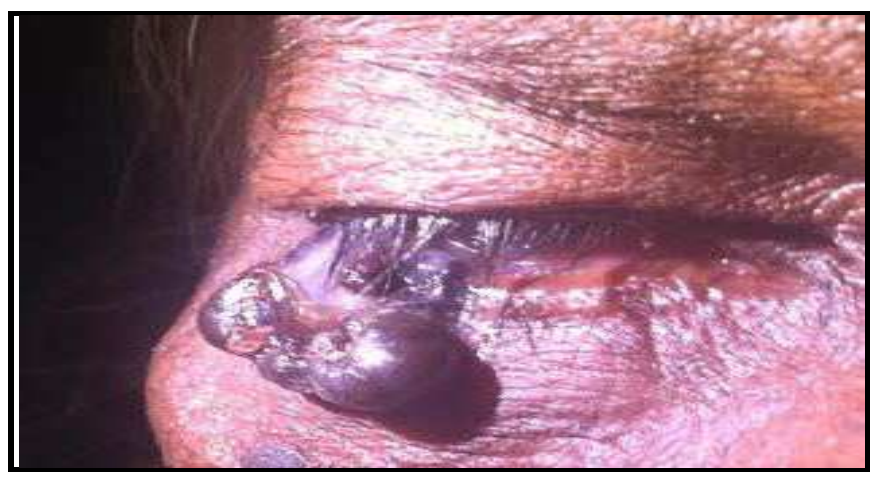

Figure 1: Clinical image showing pigmented nodule seen in the right lower eyelid near the eyelash

Head CT scan was performed but there was no involvement of the underlying structure especially bone. Regional lymph nodes did not reveal evidence of metastatic deposits. Clinically a diagnosis of malignant melanoma was made. Surgery was done under general anesthesia. Cheek rotation flap with excision was done. Tissue was sent for histopathological examination. The specimen consisted of skin covered tissue measuring $2 \mathrm{x}$ $3 \mathrm{~cm}$. External surface was skin covered with ulceroproliferative lesion. Cut surface was solid and black. (Figure 2)

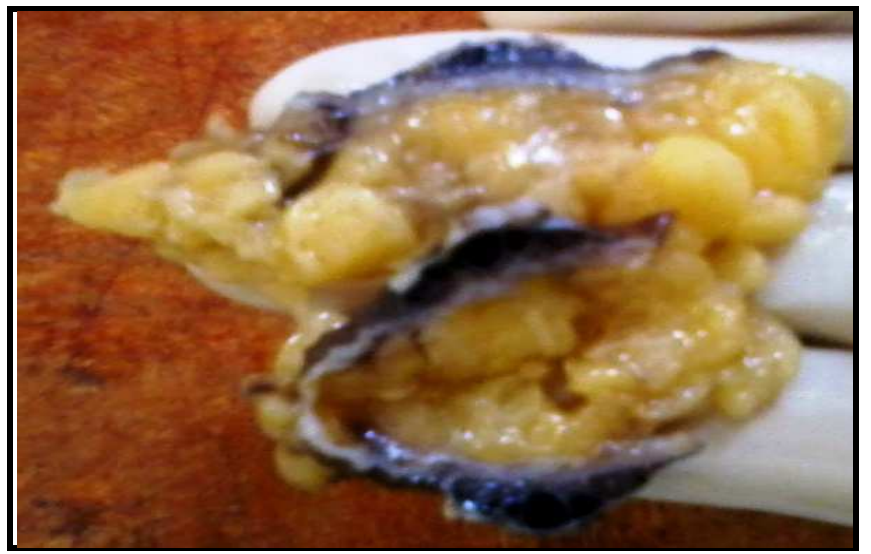

Figure 2: Cut surface of the gross specimen depicting solid tumor with black pigmentation 
Histopathology sections showed an atrophic skin with a sub epithelial tumor. (Figure $3 \mathrm{~A}$ ) The tumor was asymmetrical and composed of tumor cells arranged in lobules of varying sizes separated by fibro vascular septa. In the lobules, the tumor cells were arranged in intertwining cords as well as in tubular pattern around the central lumen which at places showed eosinophilic material which was also seen extending in between the cords of tumor cells and in the stroma. Dense melanin pigmentation was seen intracytoplasmic as well as in extracellular location. (Figure 3 B, 4 A \& 4 B) Individual cells were small oval dark with scanty cytoplasm and fine nuclear chromatin. The lobules of the tumor cells were at places seen infiltrating the underlying stroma. (Figure 5A) Atypical mitosis (> 5/10 HPF) was noted. (Figure 5 B)

Melanin bleach was done on sections which ruled out the diagnosis of malignant melanoma.

Hence a final diagnosis of low grade malignant pigmented eccrine spiradenoma was made. There was no recurrence of the tumor 1 year after surgery

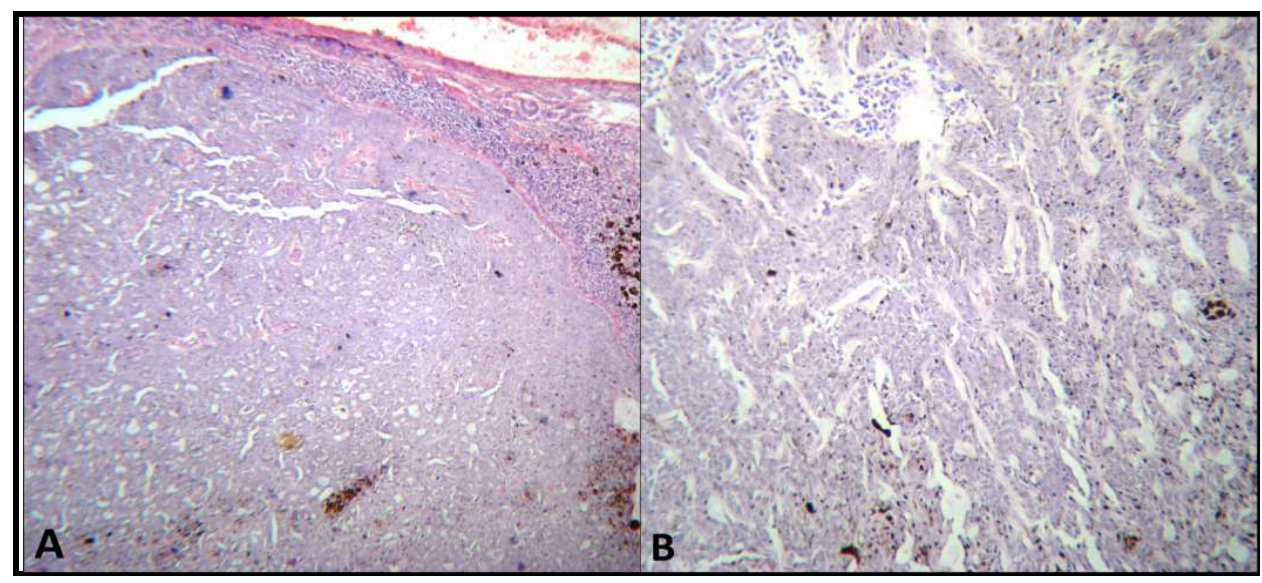

Figure 3: A: Section shows atrophic skin with a subepithelial tumor. (H \& E, $\times 40$ ) B: Section shows tumor cells arranged in intertwining cords. Extensive pigmentation is seen. (H \& E, × 40)

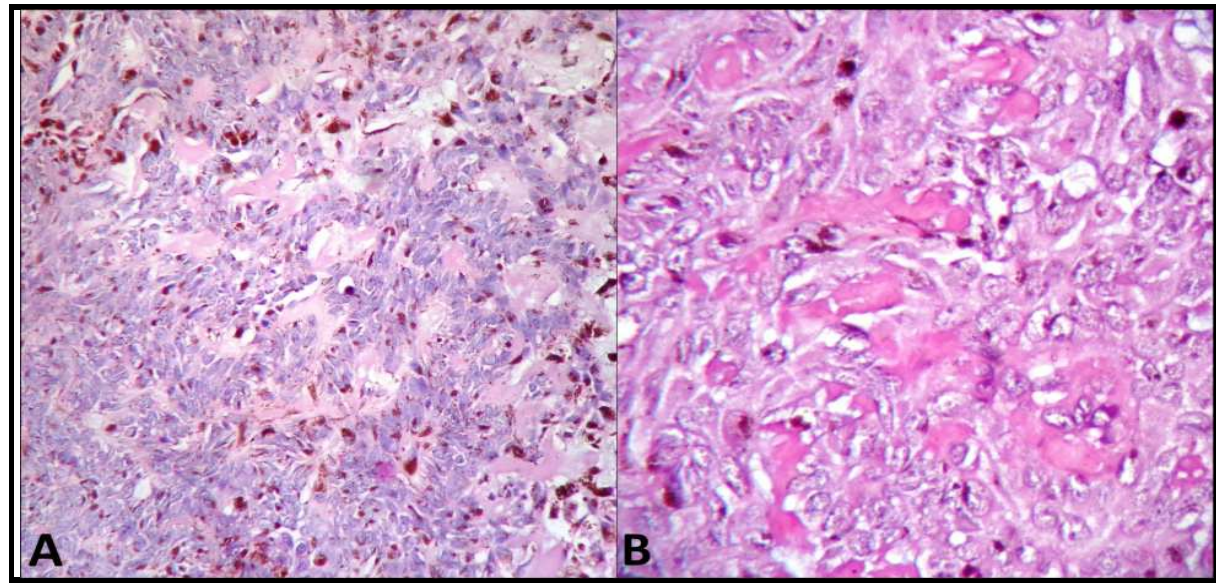

Figure 4: A: Section shows tumor cells in tubular pattern around the central lumen. (H \& E, $\times$ 100) 
B: Section shows eosinophilic basement membrane material extending in between the cords of tumor cells and in the stroma. (H \& E, x 400)

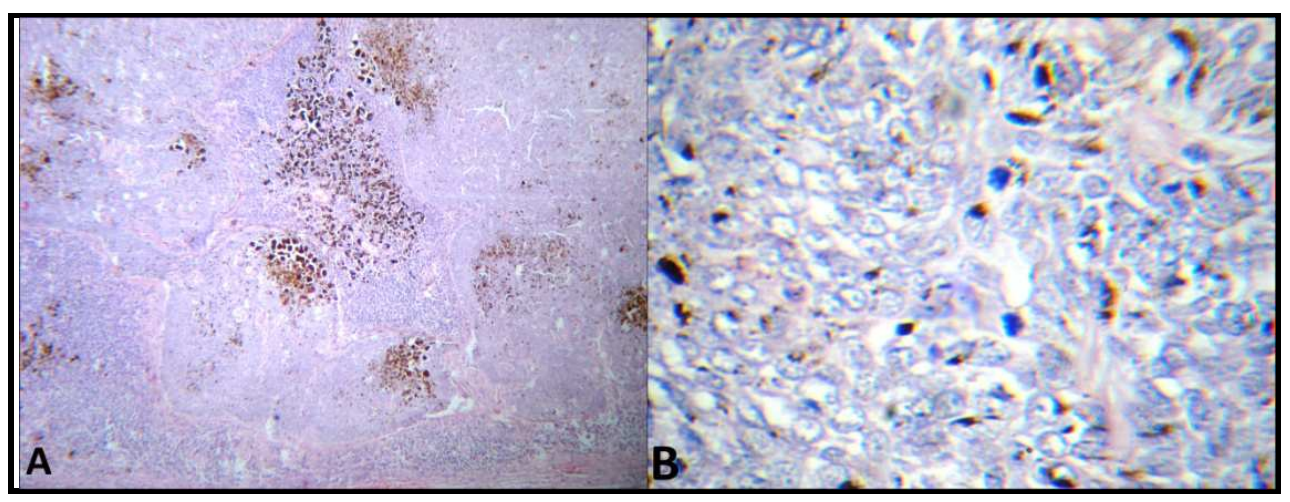

Figure 5:A: Section shows the lobules of the tumor cells infiltrating the underlying stroma. $(H \& E, \times 100)$ B: Section depicts atypical mitosis. (H \& E, ×400)

\section{Discussion}

Eyelid, specialized ocular adnexa, is a site for spectrum of tumors ranging from predominantly benign to some rare malignant tumors. Most common malignant tumor of the eyelid is basal cell carcinoma. Occasionally basal cell carcinoma may show extensive pigmentation but the phenomenon is very rare and accounts for $6 \%$ of all the basal cell carcinomas. Mechanism for pigmentation in basal cell carcinoma is explained by the proliferation of melanocytes within the tumor, leading to pigment deposition in tumor islands due to accumulation of melanin and melanophages.

Malignant adnexal tumors are as such rare and account for $0.005 \%$ of all the skin tumors. [Marenda SA and Otto RA, 1993] Malignant eccrine spiradenoma (MES) is a distinct adnexal tumor occurring predominantly in the extremities and trunk. Rarely does it occur in the head and neck region. [Beekley AC et al., 1999] Out of 102 published cases of MES, only two cases have been reported in the eyelid. [Amann J et al., 1999 and Gupta S et al., 2001] Extensive pigment deposition in MES is not a recognized entity. Clinically there is a need to differentiate this tumor from other malignant pigmented tumors of the eyelid like malignant melanoma, pigmented squamous cell carcinoma and pigmented basal cell carcinoma, in view of different treatment approach in each case. In addition to this, a variety of benign and a few malignant non melanocytic tumors of the skin present as pigmented nodules which include, benign fibrous histiocytoma, eccrine poroma, nodular hidradinoma, trichoblastoma, pilomatricoma and pilomatric carcinoma. Accurate diagnosis of these mandate histopathological examination.

Most of the time MES crop up from a preexisting benign eccrine spiradenoma, the latency period of which may extend up to 70 years. [Michael $\mathrm{T}$ et al., 2011] Clinically malignancy in a milieu of benign eccrine spiradenoma is heralded by sudden increase in the size, color change, ulceration, erythema or irritation in a previously stable lesion. [Mirza I et al., 2002]

Clinically malignant melanoma is heralded by a history of rapidly growing mass over a short duration, which is in contrast to the long history over several years followed by sudden increase in the size in case of a low grade MES, as in the our case.

The criteria for diagnosis of MES require presence of a benign spiradenoma adjacent to the malignant tumor. [Michael $\mathrm{T}$ et al., 2011] Two morphologic subtypes of MES exists, a high grade and a low grade MES. Bulk of the high grade MES is composed of carcinomatous, sarcomatous or carcinosarcomatous elements juxtaposed, 
most of the time, with classic benign eccrine spiradenoma. These tumors show hyperchromatic nucleus, frequent atypical mitosis and infiltration into the surrounding stoma. High grade MES has a poor prognosis with increased propensity for lung, brain, liver, bone and skin metastasis (after 5 months to 11 yrs of surgery) Similary presence of squamous or sarcomatous areas are also associated with poor prognosis. [Tanese K et al., 2010] However low grade MES mimics benign eccrine spiradenoma. This subtype shows monotonous population with mild pleomorphism and little mitotic activity (2 to 12 /HPF). [Leonard $\mathrm{N}$ et al., 2003]

Excessive melanin pigmentation in a low grade MES, as in our case, obscures the nuclear features, thus adding to the diagnostic dilemma. Low grade MES has better prognosis and survival as compared to their high grade counterparts. Thus these distinctions are more than semantic in nature and helps clinicians in planning accurate management. Nevertheless there are case reports of low grade MES with systemic and lymph node metastasis. [Leonard $\mathrm{N}$ et al., 2003]

Various concepts regarding the histogenesis of melanin pigmentation in a sweat gland tumor exists. Despite the fact that mature sweat gland cells lack melanoctyes and melanin pigment, the presence of melanocytes in the 14 week old acrosyringium may explain the rare occurrence of pigmentation in MES. Punia RP et al., (2008) in a case report of pigmented nodular hidradenoma, put forth that secretion of melanocyte stimulating factor by the tumor cells result in the pigmentation. [Punia RP et al., 2008] Recently, Biedener et al., in an in vivo experiment, studied the interaction of sweat gland cells with melanocytes. One of the significant findings consists of the fact that sweat gland cells can form a functional epidermal melanin unit, which clearly explains the dense pigmentation in adnexal tumors. [BottcherHaberzeth S et al., 2013]

From the data extrapolated from the 72 cases of MES, it is affirmed that wide local excision and close follow up is recommended for MES without metastatic disease. For patients with clinically palpable lymph nodes or positive sentinel lymph nodes, lymph node dissection is advocated furthermore. Due to lack of sufficient data, the role of adjuvant chemo radiotherapy remains contentious. However individualized treatment approach should be designed considering each patient's disease and morbidity. [Michael T et al., 2011]

\section{Conclusion}

The present case adds to the limited literature on pigmented adnexal tumors by reporting the first case of pigmented MES of the eyelid. Authors stress the heightened need for considering this rare tumor in the differential diagnosis of pigmented tumors of the eyelid and that all pigmented tumors of the eyelid are not melanoma.

\section{References}

1. Amann J, Spraul C. W., Mattfeld T \& Lang G. K. (1999) "Eccrinespiradenoma of the eyelid," KlinischeMonatsblätterfürAugenheilkunde,

214 (1) 53-54.

2. Beekley, A. C., Brown T. A. and Porter C. (1999) "Malignant eccrinespiradenoma: a previously unreported presentation and review of the literature,"The American Journal of Surgery, 65 (3 ) 236-40.

3. Bottcher-Haberzeth, S, Biedermann, T, Pontiggia, L, Braziulis, E, Schiestl, C, Hendriks, B, Eichhoff, O. M., Widmer, D. S, Meuli-Simmen, C, Meuli M, Reichmann E. (2013) "Human eccrine sweat gland cells turn into melanin-uptaking keratinocytes in dermo-epidermal skin substitutes," The Journal of Investigative Dermatology,133 (2) 316-24.

4. Cook, B. E. Jr \& Bartley, G. B. (2001) "Treatment options and future prospects for the management of eyelid malignancies: an evidence-based update," Ophthalmology, 108 (11) 2088-2098.

5. Gupta S, Radotra B. D, Kaur I, Handa S \& Kumar B.( 2001) "Multiple linear eccrinespiradenomas with eyelid involvement," Journal of the European 
Academy of Dermatology and Venereology, 15 (2) 163-166.

6. Leonard N, Smith D \&McNamara P. (2003) "Low

grade malignant eccrine spiradenoma with sy stemic metastases," The American Journal of Dermatopathology, 25 (3) 253-5.

7. Marenda, S. A. \& Otto, R. A. (1993) "Adnexal carcinomas of the skin," Otolaryngologic Clinics of North America, 26 (1) 87-116.

8. Michael T, Andreoli, \& Kamal M. F Itani. (2011) "Malignant eccrinespiradenoma: a meta-analysis of reported cases,"The American Journal of Surgery, 201 (5) 695-699.
9. Mirza I, Kloss R, Sieber S. C. (2002) "Malignant eccrinespiradenoma," Archives of Pathology \& Laboratory Medicine, 126 (5) 591-4.

10.Punia, R. P. , Garg, S, Bal, A \& Mohan, H.(2008) "Pigmented nodular hidradenoma masquerading as nodular malignant melanoma," Dermatology Online Journal, 14 (1) 15.

11.Tanese K, Sato T, Ishiko A.(2010) "Malignant eccrine spiradenoma: case report and review of the literature, including 15 Japanese cases", Clin Exp Dermatol,35(1)515 .. 